\title{
Adipokines, hormones related to body composition, and insulin resistance in HIV fat redistribution syndrome
}

Paula Freitas ${ }^{1 *}$, Davide Carvalho ${ }^{1}$, Ana Cristina Santos ${ }^{2,3}$, António José Madureira ${ }^{4}$, Esteban Martinez ${ }^{5}$, Jorge Pereira ${ }^{6}$, António Sarmento $^{7}$ and José Luís Medina ${ }^{1}$

\begin{abstract}
Background: Lipodystrophies are characterized by adipose tissue redistribution, insulin resistance (IR) and metabolic complications. Adipokines and hormones related to body composition may play an important role linking these alterations. Our aim was to evaluate adipocyte-derived hormones (adiponectin, leptin, resistin, TNF-a, PAI-1) and ghrelin plasma levels and their relationship with IR in HIV-infected patients according to the presence of lipodystrophy and fat redistribution.

Methods: Anthropometric and metabolic parameters, HOMA-IR, body composition by DXA and $C T$, and adipokines were evaluated in $217 \mathrm{HIV}$-infected patients on CART and 74 controls. Fat mass ratio defined lipodystrophy (L-FMR) was defined as the ratio of the percentage of the trunk fat mass to the percentage of the lower limb fat mass by DXA. Patient's fat redistribution was classified into 4 different groups according the presence or absence of either clinical lipoatrophy or abdominal prominence: no lipodystrophy, isolated central fat accumulation (ICFA), isolated lipoatrophy and mixed forms (MXF). The associations between adipokines levels and anthropometric, metabolic and body composition were estimated by Spearman correlation.

Results: Leptin levels were lower in patients with FMR-L and isolated lipoatrophy, and higher in those with ICFA and MXF. Positive correlations were found between leptin and body fat (total, trunk, leg, arm fat evaluated by DXA, and total, visceral (VAT), subcutaneous adipose tissue (SAT), and VAT/SAT ratio evaluated by CT) regardless of FMR-L, and with HOMA-IR only in patients with FMR-L. Adiponectin correlated negatively with VAT, and its mean levels were lower in patients with ICFA and higher in those with no lipodystrophy. Resistin was not correlated with adipose tissue but positively correlated with HOMA-IR in FMR-L patients. PAl-1 levels were higher in MXF-patients and their levels were positively correlated with VAT in those with FMR-L. Ghrelin was higher in HIV-infected patients than controls despite BMI-matching.
\end{abstract}

Conclusion: The overall body fat reduction in HIV lipoatrophy was associated with low leptin plasma levels, and visceral fat accumulation was mainly associated with decreased plasma levels of adiponectin.

Keywords: Lipodystrophy, HIV, Adipokines, Body composition, Insulin resistance

\footnotetext{
* Correspondence: paula_freitas@sapo.pt

${ }^{1}$ Endocrinology Department, Hospital de São João and University of Porto

Medical School, Alameda Hernâni Monteiro, 4200 Porto, Portugal

Full list of author information is available at the end of the article
} 


\section{Background}

Changes in body fat distribution are a common finding in HIV infected patients treated with combined antiretroviral therapy (cART) and this condition has many similarities to rare, congenital and acquired lipodystrophies, which are associated with depletion of subcutaneous fat, increased triglycerides and profound insulin resistance (IR) [1,2]. Adipose tissue traditionally was considered an energy storage organ, but over the last decade, it has emerged as a metabolically active endocrine organ secreting multiple bioactive peptides, collectively called "adipokines", other proteins including inflammatory mediators such as tumor necrosis factor alpha (TNF- $\alpha$ ) and plasminogen activator inhibitor-1 (PAI-1), which not only influence adipocyte function in an autocrine and paracrine fashion, but also affect more than one metabolic pathway through the bloodstream's systemic circulation [3]. All adipose tissue products seem to contribute significantly in various metabolic processes both at local and systemic levels and may induce proinflammatory state and oxidative damage, leading to initiation and progression of atherosclerosis [4]. On the other hand, these patients experience marked changes in circulating levels of adipocyte secreted hormones, including leptin and adiponectin, contributing to metabolic abnormalities, and hormones related to body composition, such as ghrelin [5]. Ghrelin is an orexigenic gut peptide that is the endogenous ligand for the growth hormone (GH) secretagogue receptor. Low ghrelin may be a marker of metabolic dysregulation and altered body fat without specifically contributing to low $\mathrm{GH}[6]$.

cART in HIV infection produces widespread metabolic complications, including hyperlipidemia, IR and lipodystrophy [peripheral fat wasting (lipoatrophy) with or without abdominal/visceral fat accumulation (lipohypertrophy)] [7]. The hormonal changes caused by increased (in lipohypertrophy) or reduced subcutaneous fat (in lipoatrophy) may be central to the metabolic abnormalities observed in HIV-lipodystrophy [6].

The aim of this study was to evaluate the relationship between fat mass, lipodystrophy defined by the Fat Mass Ratio (FMR-L) and the four different categories of fat distribution, and adipocyte derived hormones (adiponectin, leptin, resistin, TNF- $\alpha$ ), orexigenic hormones (ghrelin), prothrombotic factors (PAI-1) and IR in HIV-infected patients on cART.

\section{Methods}

\section{Subjects}

As part of a cross-sectional study, 217 consecutive clinically stable $\mathrm{HIV}$-infected adults receiving cART were evaluated between 2005 and 2008 at the Endocrinology Outpatient Department. Seventy four age-, gender- and BMI-matched HIV-uninfected controls were also evaluated. These controls were selected from a previously assembled cohort of non-institutionalized community adults, the EPI-Porto study [8]. This study was approved by the Ethics Committee for Health of Hospital São João and each patient provided written informed consent.

\section{Clinical assessment}

For each patient the following information was collected using a standardized protocol: demographic data (age, gender), duration of HIV infection, HIV infection risk factors, duration of cART and characterization of the infection. We used the "Centers for Disease Control and Prevention" (CDC) criteria for classifying the degree the infection [9]. Weight, height, circumferences of neck, waist, hip, thigh and arm were measured as previously published [10-13]. Blood pressure (BP) was measured using the established recommendations [14].

Clinical lipodystrophy was defined as a peripheral lipoatrophy with or without a central fat accumulation assessed by both patient and practitioner, as previously described [11-13]. Presence of central fat accumulation or abdominal prominence was defined by the measurement of waist circumference using the International Diabetes Federation (IDF) criteria for metabolic syndrome [15]. Patients were classified into four different categories according the presence or absence of either clinical lipoatrophy or abdominal prominence: no lipodystrophy - patients without clinical lipoatrophy and without abdominal prominence; isolated central fat accumulation - patients without clinical lipoatrophy and with abdominal prominence; isolated lipoatrophy - patients with clinical lipoatrophy and without abdominal prominence; mixed forms of lipodystrophy - patients with clinical lipoatrophy and with abdominal prominence. The clinical assessment was performed by the same practitioner (PF).

\section{Evaluation of body composition}

Body composition was assessed with whole-body dualenergy X-ray absorptiometry (DXA - Lunar Expert XL). DXA measurement was performed while the patient was in a supine position, with standard positioning of the arms and feet. Markers used in this study for trunk and lower limbs that defined regions of interest as indicated by the manufacturer. Regional fat mass values were grouped and analysed for the following anatomical regions: arms, legs, trunk and total body. The fat mass ratio (FMR) is the ratio of the percentage of the trunk fat mass to the percentage of the lower limb fat mass (FMR $=\%$ of the trunk fat mass $/ \%$ of the lower limb fat mass) [16]. We define lipodystrophy by FMR using the cut-off value of 1.961 for men and 1.329 for women [10]. The quantification of total, visceral, and peripheral abdominal fat was performed with a 64-slice computed tomography (CT) scanner (Siemens Sensation 64 Cardiac) with the same technique as previously 
described [17,18]. All values were expressed in $\mathrm{cm}^{2}$ rounded to the nearest centesimal.

\section{Laboratory analysis}

A venous blood sample was drawn after a 12-hour overnight fast. All the samples were analysed at the central laboratory of our hospital. Patients without a previous diagnosis of diabetes were submitted to a glucose tolerance test (OGTT). The test was performed as described by the World Health Organization using a glucose load containing the equivalent of $75 \mathrm{~g}$ anhydrous glucose dissolved in water.

The CD4 cell count was determined by flow cytometry and plasma HIV-1 RNA loads were measured by a quantitative reverse transcriptase polymerase chain reaction (Roche Diagnostic Systems, Inc., Branchburg, NJ, USA), which has a lower limit of detection of 50 copies $/ \mathrm{mL}$.

\section{Adipokines and hormones related to body composition} Leptin (Human Leptin RIA Kit, Linco Research), adiponectin (Human Adiponectin RIA Kit, Linco Research), resistin (Human Resistin ELISA Kit, Linco Research), TNF- $\alpha$ (TNF- $\alpha$ IRMA Kit, BioSource), ghrelin (Ghrelin Active RIA Kit, Linco Research) and PAI-1 (Human PAI-1 ELISA Kit, IBL International GMBH) were measured in the Nobre Laboratory of the Porto Medical School. For leptin, the intra-assay precision is $4.6 \%$ and inter-assay precision $5.0 \%$. For adiponectin, the intraassay precision is $1.8 \%$ and inter-assay precision $9.3 \%$. For resistin, the intra-assay precision is $3.2 \%$ and interassay precision $7.1 \%$. For TNF- $\alpha$, the intra-assay precision is $3.1 \%$ and inter-assay precision $5.7 \%$. For ghrelin, the intra-assay precision is $6.7 \%$ and inter-assay precision $9.6 \%$. The overall intra-assay coefficient of variation for PAI-1 was $4.7 \%$.

\section{Insulin resistance evaluation}

Insulin resistance was defined by the homeostasis model assessment of insulin resistance (HOMA) and insulin sensitivity by the quantitative insulin sensitivity check index (QUICKI). These indexes were calculated by the following formulas:

HOMA-IR index $=($ insulin $0 \times$ glucose 0$) / 22.5$ [19]

QUICKI $=1 /[\log$ (fasting insulin in $\mathrm{mU} / \mathrm{l})+\log$ (fasting plasma glucose in $\mathrm{mg} / \mathrm{dL}$ )] [20].

Glucose was expressed in $\mathrm{mmol} / \mathrm{L}$ and insulin in $\mu \mathrm{UI} / \mathrm{mL}$.

\section{Statistical analysis}

Data were described as mean and standard deviation (SD) for quantitative variables and compared using the Student-t test or the Mann-Whitney test as appropriate. For the comparison between the four groups of fat distribution and adipokines parameters the Kruskall-Wallis test was used. Categorical variables were described as counts and proportions, and compared using the chisquare or Fisher's exact test.

For estimating the association between adipokines levels and anthropometric, metabolic and body composition, Spearman correlation coefficients were calculated. Statistical analysis was performed using the SPSS version 18.0 software (SPSS Inc., Chicago, Illinois, USA). All probabilities were two tailed and $\mathrm{p}$ values $<0.05$ were regarded as significant, with the exception of the correlations between adipokines levels and anthropometric, metabolic and body composition, considering $\mathrm{p}$ values $<0.001$ as significant after applying the Bonferroni correction.

\section{Results}

Sample characteristics, adipokines and hormones related to body composition in HIV-infected patients and HIV-uninfected controls

HIV-infected patients had significantly higher waist/hip ratio, glucose, triglyceride, insulin, HOMA, and ghrelin levels, and adiponectin/leptin ratio. However, they had lower weight, hip and arm circumferences, HDL-cholesterol levels, QUICKI, resistin, adiponectin and leptin levels. No differences were observed between HIV-infected patients and HIV-uninfected controls regarding waist circumference, systolic and diastolic blood pressure and total cholesterol, LDL-cholesterol, uric acid, hsCRP and TNF- $\alpha$ levels (Table 1).

\section{Adipokines and hormones related to body composition in} HIV-infected patients according to gender

HIV-infected females had higher hip circumference, total fat mass and subcutaneous adipose tissue (SAT) on abdominal CT scan evaluation and total, trunk, leg and arm mass fat by DXA. They also had lower visceral adipose tissue (VAT) and VAT/SAT ratio on CT scan. Also, females had higher leptin levels and lower TNF- $\alpha$ and adiponectin/leptin ratio. No gender-related differences were observed in adiponectin, resistin, ghrelin and PAI-1 levels (Table 2).

\section{Adipokines and hormones related to body composition according to the four categories of body composition} Patients with isolated central fat accumulation and mixed forms of lipodystrophy were older and had higher BMI. Patients with no lipodystrophy had higher levels of adiponectin and those with isolated central fat accumulation had the lowest levels of adiponectin. The levels of leptin were higher in patients with isolated central fat accumulation and mixed forms of lipodystrophy and lower in those with isolated lipoatrophy. No significant differences were observed in resistin, ghrelin and TNF- $\alpha$ levels between the four groups of body composition. Patients with no lipodystrophy and mixed forms of lipodystrophy had higher levels of PAI-1, and the lowest levels of PAI-1 were 
Table 1 Sample characteristics, adipokines and hormones related to body composition in HIV-uninfected controls and HIV-infected patients

\begin{tabular}{|c|c|c|c|}
\hline & HIV-uninfected controls & HIV-infected patients & $\mathrm{p}$ \\
\hline$n$ & 74 & 217 & \\
\hline Age [years, mean (sd)] & $47.2(9.7)$ & $46.6(11.6)$ & 0.73 \\
\hline \multicolumn{4}{|l|}{ Gender $[n(\%)]$} \\
\hline Male & $40(54.1)$ & $144(66.9)$ & \\
\hline Female & $34(45.9)$ & $73(33.6)$ & 0.08 \\
\hline Weight $[\mathrm{Kg}$, mean $(\mathrm{sd})]$ & $71.5(13.4)$ & $67.8(12.3)$ & 0.03 \\
\hline BMI $\left[\left(\mathrm{kg} / \mathrm{m}^{2}\right)\right.$, mean $\left.(\mathrm{sd})\right]$ & $26.3(4.3)$ & $35.1(4.5)$ & 0.05 \\
\hline Waist circumference [cm, mean (sd)] & $90.7(11.6)$ & $91.2(11.6)$ & 0.75 \\
\hline Hip circumference [cm, mean (sd)] & 99.8. (7.1) & $94.5(8.4)$ & $<0.001$ \\
\hline Waist/hip ratio [mean (sd)] & $0.91(0.08)$ & $0.96(0.08)$ & $<0.001$ \\
\hline Arm circumference $[\mathrm{cm}$, mean (sd)] & $30.6(3.5)$ & $27.0(3.0)$ & $<0.001$ \\
\hline Systolic blood pressure [mmHg, mean (sd)] & $122.2(17.3)$ & $121.9(17.5)$ & 0.99 \\
\hline Diastolic blood pressure [mmHg, mean (sd)] & $78.9(10.1)$ & $76.7(11.3)$ & 0.24 \\
\hline Glucose $[\mathrm{mg} / \mathrm{dL}$, mean $(\mathrm{sd})]$ & $91.7(12.2)$ & $107.7(41.5)$ & 0.03 \\
\hline Insulin $[\mu \mathrm{U} / \mathrm{mL}$, mean $(\mathrm{sd})]$ & $5.5(4.9)$ & $11.2(10.6)$ & $<0.001$ \\
\hline HOMA [mean (sd)] & $1.3(1.4)$ & $3.1(3.2)$ & $<0.001$ \\
\hline QUICKI [mean (sd)] & $0.39(0.05)$ & $0.36(0.08)$ & $<0.001$ \\
\hline Total cholesterol [mg/dL, mean (sd)] & $217.2(43.6)$ & $224.2(58.0)$ & 0.26 \\
\hline LDL cholesterol [mg/dL, mean (sd)] & $134.9(38.8)$ & $128.5(49.9)$ & 0.42 \\
\hline HDL cholesterol [mg/dL, mean (sd)] & $57.4(14.9)$ & $46.1(13.6)$ & $<0.001$ \\
\hline Triglycerides [mg/dL, mean (sd)] & $119.8(57.6)$ & $267.5(182.8)$ & $<0.001$ \\
\hline Uric acid [mg/dL, mean (sd)] & $49.6(15.4)$ & $46.8(15.0)$ & 0.165 \\
\hline hs-CRP [mg/dL, mean (sd)] & $0.36(0.54)$ & $0.45(0.73)$ & 0.55 \\
\hline Resistin [ng/mL, mean (sd)] & $15.5(11.0-22.2)$ & $10.4(8.0-13.9)$ & $<0.001$ \\
\hline Adiponectin $[\mu \mathrm{g} / \mathrm{mL}$, mean (sd)] & $5.18(2.80-7.74)$ & $2.68(1.19-5.46)$ & $<0.001$ \\
\hline TNF-a [pg/mL, mean (sd)] & $45.3(29.9-60.5)$ & $44.6(30.1-73.6)$ & 0.63 \\
\hline Ghrelin [pg/mL, mean (sd)] & $18.6(10.5-25.4)$ & $75.0(47.7-139.5)$ & $<0.001$ \\
\hline Leptin [ng/mL, mean (sd)] & $11.8(6.3-21.3)$ & $3.4(1.9-7.6)$ & $<0.001$ \\
\hline Adiponectin/leptin ratio [mean (sd)] & $1.00(1.95)$ & $2.29(5.87)$ & 0.02 \\
\hline
\end{tabular}

observed in those with isolated central fat accumulation. The adiponectin/leptin ratio was higher in patients with no lipodystrophy and lower in patients with isolated central fat accumulation, followed by the patients with mixed forms of lipodystrophy (Table 3).

Sample characteristics, body composition, metabolic parameters, adipokines and hormones related to body composition, according to the presence lipodystrophy defined by FMR

In this sample of $217 \mathrm{HIV}-1$ infected patients (144 men and 73 women) on cART, $41 \%$ of patients presented lipodystrophy defined by FMR (FMR-L). Table 4 shows the characteristics of the study sample according to the presence of FMR-L. Patients with FMR-L were more frequently male, older and had a longer duration of HIV infection and length of cART. No significant differences were found in weight, BMI, waist, arm and thigh circumferences among patients with or without FMR-L. Patients with FMR-L had a higher neck circumference and waist/ hip ratio, but a lower hip circumference. Also, they had significantly a higher mean CD4+ cell count, but no significant difference in viral suppression rate was observed. No differences were observed between patients with and without FMR-L regarding CART regimens and CDC classification. Also, no differences were observed regarding diastolic blood pressure, hs-CRP, total cholesterol, LDL and HDL cholesterol levels. The patients with FMR-L had higher systolic blood pressure, uric acid, triglycerides, HOMA, fasting glucose and insulin levels. Regarding 
Table 2 Body composition, adipokines and hormones related to body composition in HIV-infected patients, according to gender

\begin{tabular}{|c|c|c|c|}
\hline & Male & Female & $\mathbf{P}$ \\
\hline$n$ & 144 & 73 & \\
\hline Age [years, mean (SD)] & $46.8(11.2)$ & $46.2(12.5)$ & 0.72 \\
\hline $\mathrm{BMI}\left[\left(\mathrm{kg} / \mathrm{m}^{2}\right)\right.$, mean $\left.(\mathrm{SD})\right]$ & $24.7(3.8)$ & $26.1(5.6)$ & 0.05 \\
\hline Waist circumference $[\mathrm{cm}$, mean (SD)] & $91.2(10.6)$ & $91.2(13.5)$ & 0.98 \\
\hline Hip circumference [cm, mean (SD)] & $93.1(6.7)$ & $97.2(10.5)$ & 0.003 \\
\hline Total fat mass $C T\left[\mathrm{~cm}^{3}\right.$, mean $\left.(\mathrm{SD})\right]$ & $243.4(136.2)$ & $356.0(166.7)$ & $<0.001$ \\
\hline VAT CT $\left[\mathrm{cm}^{3}\right.$, mean (SD)] & $140.7(86.2)$ & $113.6(69.9)$ & 0.02 \\
\hline SAT CT $\left[\mathrm{cm}^{3}\right.$, mean (SD)] & $102.6(76.9)$ & $242.4(125.7)$ & $<0.001$ \\
\hline VAT/SAT ratio & $2.2(2.4)$ & $0.55(0.40)$ & $<0.001$ \\
\hline Total fat mass DXA [\%, mean (SD)] & $16.8(8.1)$ & $33.3(10.0)$ & $<0.001$ \\
\hline Trunk fat in DXA [\%, mean (SD)] & $20.6(9.8)$ & $33.4(10.3)$ & $<0.001$ \\
\hline Leg fat mass DXA [\%, mean (SD)] & $12.2(9.1)$ & $31.9(12.5)$ & $<0.001$ \\
\hline Arm fat mass DXA [\%, mean (SD)] & $15.2(8.9)$ & $42.3(11.4)$ & $<0.001$ \\
\hline Adiponectin [ $\mathrm{\mu g} / \mathrm{mL}$, median (25th and 75th percentile)] & $3.34(1.79-6.59)$ & $3.00(1.36-6.55)$ & 0.39 \\
\hline Leptin [ng/mL, median (25th and 75th percentile)] & $3.0(1.8-5.8)$ & $11.2(5.7-18.0)$ & $<0.001$ \\
\hline Resistin $[\mathrm{ng} / \mathrm{mL}$, median (25th and 75th percentile)] & $11.2(8.4-17.0)$ & $11.2(8.9-16.8)$ & 0.42 \\
\hline Ghrelin [pg/mL, median (25th and 75th percentile)] & $55.5(26.9-99.2)$ & $44.0(21.4-79.2)$ & 0.07 \\
\hline PAl-1 [pg/mL, median (25th and 75th percentile)] & $61.3(38.5-104.3)$ & $43.4(30.8-95.8)$ & 0.37 \\
\hline TNF-a [pg/mL, median (25th and 75th percentile)] & $49.9(31.6-72.4)$ & $39.7(27.2-60.0)$ & 0.03 \\
\hline Adiponectin/leptin ratio, median (25th and 75th percentile) & $0.945(0.34-2.04)$ & $0.245(0.09-0.63)$ & $<0.001$ \\
\hline
\end{tabular}

OGTT, glucose and insulin at 2 hours were higher in patients with FMR-L and as expected, QUICKI was lower in patients with FMR-L.

On DXA evaluation, patients with FMR-L had lower total, leg and arm fat mass (either in \% or Kg) than patients without FMR-L with no significant difference in trunk fat mass. On CT evaluation at abdominal level, patients with FMR-L had higher VAT and lower SAT with a higher central/peripheral fat ratio, with no difference in total fat at this level.

Leptin levels were lower in patients with FMR-L. No significant differences were observed in levels of adiponectin,

Table 3 Adipokines and hormones related to body composition according to four categories of body composition

\begin{tabular}{|c|c|c|c|c|c|}
\hline & $\begin{array}{l}\text { No lipodystrophy } \\
\mathrm{N}=\mathbf{2 3}\end{array}$ & $\begin{array}{l}\text { Isolated central fat } \\
\text { accumulation } \mathrm{N}=56\end{array}$ & $\begin{array}{c}\text { Isolated } \\
\text { lipoatrophy } \mathrm{N}=78\end{array}$ & $\begin{array}{c}\text { Mixed forms of } \\
\text { lipodystrophy } N=60\end{array}$ & $\mathbf{P}$ \\
\hline Age [years, mean (SD)] & $39.9(11.3)$ & $46.5(12.6)$ & $44.1(9.7)$ & $52.6(10.8)$ & $<0.001$ \\
\hline BMI $\left[\mathrm{kg} / \mathrm{m}^{2}\right.$, mean $\left.(\mathrm{SD})\right]$ & $23.0(2.4)$ & $29.1(4.4)$ & $21.9(2.8)$ & $26.5(3.2)$ & $<0.001$ \\
\hline $\begin{array}{l}\text { Adiponectin }[\mu \mathrm{g} / \mathrm{mL} \text {, median ( } 25 \text { th and } \\
75 \text { th percentile)] }\end{array}$ & $4.40(2.43-8.75)$ & $1.90(0.89-3.48)$ & $2.64(1.20-6.53)$ & $2.81(1.04-5.22)$ & 0.02 \\
\hline Leptin [ng/mL, median (25th and 75th percentile)] & $3.0(2.0-5.7)$ & $10.3(5.3-15.1)$ & $2.1(1.4-3.3)$ & $4.3(2.4-7.0)$ & $<0.001$ \\
\hline $\begin{array}{l}\text { Resistin }[\mathrm{ng} / \mathrm{mL} \text {, median }(25 \text { th and } \\
\text { 75th percentile)] }\end{array}$ & $9.1(7.9-12.6)$ & $10.3(7.2-12.0)$ & $9.5(8.1-15.3)$ & $11.2(8.1-16.4)$ & 0.36 \\
\hline $\begin{array}{l}\text { Ghrelin }[\mathrm{pg} / \mathrm{mL} \text {, median (25th and } \\
\text { 75th percentile)] }\end{array}$ & $72.0(52.4-110.3)$ & $71.0(41.3-110.8)$ & $81.9(46.2-194.3)$ & $79.7(52.4-122.4)$ & 0.42 \\
\hline PAl-1 [pg/mL, median (25th and 75th percentile)] & $111.9(64.8-162.8)$ & $40.4(30.6-45.8)$ & $61.3(28.3-96.4)$ & $68.7(34.6-109.6)$ & 0.047 \\
\hline TNF-a [pg/mL, median (25th and 75th percentile)] & $42.8(33.1-60.5)$ & $41.6(27.7-83.4)$ & $49.8(30.7-72.6)$ & 43.7 (29.6-79.8) & 0.91 \\
\hline $\begin{array}{l}\text { Adiponectin/leptin ratio median (25th and } \\
\text { 75th percentile) }\end{array}$ & $1.30(0.97-2.50)$ & $0.19(0.06-0.38)$ & $1.17(0.43-4.60)$ & $0.73(0.20-1.43)$ & $<0.001$ \\
\hline
\end{tabular}


Table 4 Sample characteristic, body composition, adipokines and hormones related to body composition according to the presence of lipodystrophy defined by FMR

\begin{tabular}{|c|c|c|c|}
\hline & Without lipodystrophy & With lipodystrophy & $\mathbf{P}$ \\
\hline n (\%) & $128(59.0)$ & $89(41.0)$ & - \\
\hline \multicolumn{4}{|l|}{ Gender $[\mathrm{n}(\%)]$} \\
\hline Male & $76(59.4)$ & $68(76.4)$ & \\
\hline Female & $52(40.6)$ & $21(23.6)$ & 0.01 \\
\hline Age [years, mean (sd)] & $44.7(12.2)$ & $49.4(10.2)$ & 0.003 \\
\hline Duration of HIV infection [years, mean (sd)] & $7.4(3.9)$ & $8.6(3.6)$ & 0.007 \\
\hline cART [years, mean (sd)] & $5.8(3.8)$ & $8.0(3.4)$ & $<0.001$ \\
\hline Weight [Kg, mean (sd)] & $67.4(13.0)$ & $68.3(11.1)$ & 0.60 \\
\hline BMI [(kg/m²), mean $(\mathrm{sd})]$ & $25.0(4.8)$ & $25.3(4.5)$ & 0.68 \\
\hline Waist circumference [cm, mean (sd)] & $90.2(12.2)$ & $92.7(10.5)$ & 0.10 \\
\hline Hip circumference [cm, mean (sd)] & $95.9(9.2)$ & $92.5(6.7)$ & 0.002 \\
\hline Thigh circumference [cm, mean (sd)] & $48.3(5.6)$ & $46.9(4.8)$ & 0.07 \\
\hline Arm circumference $[\mathrm{cm}$, mean (sd)] & $26.7(3.2)$ & $27.2(2.7)$ & 0.28 \\
\hline Neck circumference $[\mathrm{cm}$, mean (sd)] & $36.4(3.8)$ & $37.9(3.7)$ & 0.01 \\
\hline Waist/hip circumference ratio & $0.94(0.08)$ & $1.00(0.07)$ & $<0.001$ \\
\hline CD4 cell count $\left[\right.$ cells $/ \mathrm{mm}^{3}$, mean (sd)] & $498.9(289.4)$ & $644.6(354.1)$ & 0.001 \\
\hline HIV RNA $(<50)[n(\%)]$ & $109(85.2)$ & $81(91.0)$ & 0.45 \\
\hline \multicolumn{4}{|l|}{ HIV risk factor [n (\%)] } \\
\hline Injecting drug user & $36(28.6)$ & $17(19.1)$ & \\
\hline Homosexual contact & $7(5.6)$ & $16(18.0)$ & \\
\hline Heterosexual contact & $81(64.3)$ & $54(60.7)$ & \\
\hline Others & $2(1.6)$ & $2(2.2)$ & 0.02 \\
\hline \multicolumn{4}{|l|}{ CDC [n (\%)] } \\
\hline A & $67(52.8)$ & $57(64.0)$ & 0.16 \\
\hline B & $2(1.6)$ & $0(0)$ & \\
\hline C & $58(45.7)$ & $32(36.0)$ & \\
\hline \multicolumn{4}{|l|}{ ART [n (\%)] } \\
\hline IP & $65(52.0)$ & $48(53.9)$ & 0.89 \\
\hline NNRTI & $62(49.6)$ & $45(50.6)$ & 0.89 \\
\hline NRTI & $121(96.8)$ & $86(96.6)$ & 1.00 \\
\hline Total Cholesterol [mg/dL, mean (sd)] & $218.1(56.4)$ & $233.1(59.5)$ & 0.06 \\
\hline LDL-cholesterol [mg/dL, mean (sd)] & $125.4(50.2)$ & $133.0(49.4)$ & 0.27 \\
\hline HDL- cholesterol [mg/dL, mean (sd)] & $47.2(14.6)$ & $44.4(11.7)$ & 0.14 \\
\hline Triglycerides [mg/dL, mean (sd)] & $244.8(117.6)$ & $300.1(195.3)$ & 0.03 \\
\hline Uric acid $[\mathrm{mg} / \mathrm{L}$, mean $(\mathrm{sd})]$ & $43.8(14.3)$ & $51.0(15.1)$ & $<0.001$ \\
\hline Systolic blood pressure [mmHg, mean (sd)] & $120.0(18.6)$ & $124.8(15.6)$ & 0.04 \\
\hline Diastolic blood pressure [mmHg, mean (sd)] & $75.7(11.8)$ & $78.1(10.5)$ & 0.14 \\
\hline Glucose 0 [mg/dL, mean $(\mathrm{sd})]$ & $103.0(39.5)$ & $114.4(43.7)$ & 0.002 \\
\hline Glucose $2 \mathrm{~h}$ at OGTT [mg/dL, mean (sd)] & $123.9(42.9)$ & $139.7(49.0)$ & 0.03 \\
\hline Insulin $0[\mu \mathrm{U} / \mathrm{mL}$, mean (sd)] & $9,96(10.50)$ & $12.96(10.55)$ & 0.005 \\
\hline Insulin $2 \mathrm{~h}$ at $\mathrm{OGTT}[\mu \mathrm{U} / \mathrm{mL}$, mean (sd)] & 49. $8(40.1)$ & $88.3(142.2)$ & 0.005 \\
\hline HOMA [mean (sd)] & $2.65(3.01)$ & $3.70(3.28)$ & $<0.001$ \\
\hline QUICKI [mean (sd)] & $0.37(0.10)$ & $0.34(0.4)$ & $<0.001$ \\
\hline
\end{tabular}


Table 4 Sample characteristic, body composition, adipokines and hormones related to body composition according to the presence of lipodystrophy defined by FMR (Continued)

\begin{tabular}{|c|c|c|c|}
\hline hsCRP [mg/dL, mean (sd)] & $0.50(0.90)$ & $0.36(0.27)$ & 0.28 \\
\hline Adiponectin [ $\mathrm{gg} / \mathrm{mL}$, mean (sd)] & $5.64(7.39)$ & $3.95(4.49)$ & 0.23 \\
\hline Leptin [ng/mL, mean (sd)] & $7.3(8.5)$ & $3.9(3.3)$ & 0.008 \\
\hline Resistin [ng/mL, mean (sd)] & $12.8(13.4)$ & $12.8(7.4)$ & 0.37 \\
\hline Ghrelin[pg/mL, mean (sd)] & $117.4(133.6)$ & $118.1(106.7)$ & 0.45 \\
\hline PAl-1 [pg/mL, mean (sd)] & $62.6(43.6)$ & $102.1(110.1)$ & 0.26 \\
\hline TNF-a $[\mathrm{pg} / \mathrm{mL}$, mean (sd)] & $61.9(50.3)$ & $52.3(33.9)$ & 0.43 \\
\hline Adiponectin/leptin ratio [mean (sd)] & $2.72(7.21)$ & $1.69(3.13)$ & 0.42 \\
\hline \multicolumn{4}{|l|}{ Fat mass $[\%$, mean (sd)] DXA } \\
\hline Total & $24.8(13.1)$ & $19.3(8.5)$ & 0.002 \\
\hline Trunk & $25.7(13.3)$ & $24.3(9.0)$ & 0.53 \\
\hline Leg & $24.4(14.7)$ & $11.1(7.4)$ & $<0.001$ \\
\hline Arm & $27.2(17.1)$ & $20.3(13.6)$ & 0.001 \\
\hline \multicolumn{4}{|l|}{ Fat mass $[\mathrm{Kg}$, mean $(\mathrm{sd})] \mathrm{DXA}$} \\
\hline Total & $17.3(11.1)$ & $13.2(7.3)$ & 0.02 \\
\hline Trunk & $9.3(6.0)$ & $9.0(4.8)$ & 0.69 \\
\hline Leg & $5.2(3.6)$ & $2.1(1.6)$ & $<0.001$ \\
\hline Arm & $2.1(1.9)$ & $1.5(1.2)$ & 0.008 \\
\hline \multicolumn{4}{|l|}{ Body Fat Mass by Quantitative CT } \\
\hline Total $\left[\mathrm{cm}^{2}\right.$, mean $\left.(\mathrm{sd})\right]$ & $282.9(174.9)$ & $276.5(126.6)$ & 0.92 \\
\hline VAT $\left[\mathrm{cm}^{2}\right.$, mean $\left.(\mathrm{sd})\right]$ & $111.2(85.4)$ & $139.4(68.7)$ & $<0.001$ \\
\hline SAT $\left[\mathrm{cm}^{2}\right.$, mean $\left.(\mathrm{sd})\right]$ & $171.7(125.9)$ & $117.0(92.4)$ & 0.001 \\
\hline VAT/SAT ratio [mean (sd)] & $1.07(1.46)$ & $2.44(2.60)$ & $<0.001$ \\
\hline
\end{tabular}

Note: CART - combined antiretroviral therapy; BMI. Body mass index; OGTT- oral glucose tolerance test; DXA - dual-energy X-ray absorptiometry CT - computed tomography; VAT- visceral adipose tissue; SAT - subcutaneous adipose tissue.

resistin, ghrelin, PAI-1, TNF- $\alpha$ and adiponectin/leptin ratio among patients with or without FMR-L (Table 4).

\section{Adipokines, body composition hormones and metabolic parameters}

We evaluated the correlations between leptin, resistin, TNF- $\alpha$ and adiponectin with age, waist/hip circumference ratio, arm, waist, hip circumferences, BMI, fasting glucose and insulin, HOMA, triglycerides and total cholesterol in all the HIV-infected patients and HIVuninfected controls. Leptin was positively associated with arm, waist, hip circumferences, BMI and fasting insulin both in the HIV-infected patients and in HIVuninfected controls. In HIV-uninfected controls, leptin was also positively correlated with HOMA. A negative correlation was observed between resistin and hip circumference and BMI in the HIV-infected patients, while a positive correlation between resistin and triglycerides was found in the HIV-uninfected controls. Also, a negative correlation was observed between TNF- $\alpha$ and total cholesterol in HIV-infected patients, and between adiponectin and arm circumference in controls (Table 5).
Regarding the effect of lipodystrophy on the association between anthropometric and metabolic parameters in HIV-infected patients, a significant positive correlation was found between leptin and: insulin at 2 hours on OGTT; total, trunk, leg, arm fat evaluated by DXA; and total, SAT, VAT/SAT ratio on abdominal level evaluated by CT scan in these patients, regardless of the presence of FMR-L. Leptin was also positively associated with arm, thigh, waist, hip circumferences, BMI, glucose at 2 hours on OGTT, total cholesterol, and was negatively associated with length of cART in patients without FMR-L. Patients with FMR-L also exhibited positive associations between resistin levels and duration of HIVinfection, as well as between PAI-1 and VAT. A negative association was found between adiponectin and arm fat mass measured by DXA in FMR-L patients. A positive correlation was observed between TNF- $\alpha$, fasting glucose and A1c in patients without FMR-L (Table 6).

The adiponectin/leptin ratio was not associated with IR [HOMA $(\mathrm{r}=-0.148, \mathrm{p}=0.07)$ or QUICKI $(\mathrm{r}=0.148$, $\mathrm{p}=0.07)]$ in overall HIV patients and in male patients [HOMA $(\mathrm{r}=-0.138, \mathrm{p}=0.16)$ or QUICKI $(\mathrm{r}=0.126$, 
Table 5 Associations between adipokines and anthropometric and metabolic parameters in HIV patients and controls

\begin{tabular}{|c|c|c|c|c|c|c|c|c|c|c|c|c|c|c|c|c|}
\hline \multirow{4}{*}{$\begin{array}{l}\text { Correlation } \\
\text { between }\end{array}$} & \multicolumn{4}{|c|}{ Leptin } & \multicolumn{4}{|c|}{ Resistin } & \multicolumn{4}{|c|}{ TNF- $a$} & \multicolumn{4}{|c|}{ Adiponectin } \\
\hline & \multirow{2}{*}{\multicolumn{2}{|c|}{$\begin{array}{c}\begin{array}{c}\text { Total of HIV } \\
\text { patients }\end{array} \\
n=161\end{array}$}} & \multirow{2}{*}{\multicolumn{2}{|c|}{$\begin{array}{l}\text { Controls } \\
\qquad n=67\end{array}$}} & \multirow{2}{*}{\multicolumn{2}{|c|}{$\begin{array}{c}\begin{array}{c}\text { Total of HIV } \\
\text { patients }\end{array} \\
n=170\end{array}$}} & \multirow{2}{*}{\multicolumn{2}{|c|}{$\begin{array}{c}\text { Controls } \\
n=74\end{array}$}} & \multirow{2}{*}{\multicolumn{2}{|c|}{$\begin{array}{c}\begin{array}{c}\text { Total of HIV } \\
\text { patients }\end{array} \\
n=160\end{array}$}} & \multirow{2}{*}{\multicolumn{2}{|c|}{$\begin{array}{c}\text { Controls } \\
n=74\end{array}$}} & \multirow{2}{*}{\multicolumn{2}{|c|}{$\begin{array}{c}\begin{array}{c}\text { Total of HIV } \\
\text { patients }\end{array} \\
n=159\end{array}$}} & \multirow{2}{*}{\multicolumn{2}{|c|}{$\begin{array}{c}\text { Controls } \\
n=74\end{array}$}} \\
\hline & & & & & & & & & & & & & & & & \\
\hline & $r$ & $\mathbf{P}$ & $\mathbf{R}$ & $\mathbf{p}$ & $r$ & $p$ & $r$ & $p$ & $\mathbf{R}$ & $p$ & $r$ & $p$ & $r$ & $\mathbf{P}$ & $r$ & $\mathbf{P}$ \\
\hline Age & 0.05 & 0.55 & -0.10 & 0.44 & -0.02 & 0.84 & -0.15 & 0.21 & -0.007 & 0.92 & -0.27 & 0.02 & -0.05 & 0.52 & -0.03 & 0.78 \\
\hline Wais/hip ratio & 0.09 & 0.24 & 0.10 & 0.41 & 0.05 & 0.52 & 0.13 & 0.27 & 0.06 & 0.44 & 0.08 & 0.51 & -0.09 & 0.23 & -0.11 & 0.34 \\
\hline Waist circumference & 0.43 & $<0.001 *$ & 0.36 & 0.003 & -0.08 & 0.27 & 0.07 & 0.57 & 0.05 & 0.48 & 0.08 & 0.49 & -0.1 & 0.19 & -0.16 & 0.1 \\
\hline Hip circumference & 0.41 & $<0.001^{*}$ & 0.54 & $<0.001 *$ & -0.15 & 0.04 & 0.03 & 0.78 & 0.005 & 0.95 & 0.1 & 0.41 & -0.05 & 0.52 & -0.11 & 0.36 \\
\hline Arm circumference & 0.29 & $<0.001 *$ & 0.33 & 0.007 & -0.11 & 0.21 & -0.008 & 0.95 & -0.05 & 0.47 & 0.04 & 0.72 & -0.09 & 0.29 & -0.24 & 0.04 \\
\hline BMl & 0.51 & $<0.001 *$ & 0.49 & $<0.001^{*}$ & -0.17 & 0.02 & 0.01 & 0.92 & 0.05 & 0.52 & 0.12 & 0.32 & -0.06 & 0.42 & -0.18 & 0.1 \\
\hline Glucose at 0 & -0.02 & 0.84 & 0.03 & 0.85 & 0.11 & 0.16 & -0.04 & 0.74 & 0.10 & 0.17 & -0.20 & 0.09 & 0.04 & 0.58 & -0.07 & 0.58 \\
\hline Insulin at 0 & 0.17 & 0.03 & 0.35 & 0.005 & 0.12 & 0.12 & 0.09 & 0.46 & 0.04 & 0.57 & 0.21 & 0.08 & -0.12 & 0.11 & 0.02 & 0.87 \\
\hline HOMA & 0.13 & 0.11 & 0.31 & 0.02 & 0.15 & 0.06 & 0.04 & 0.78 & 0.05 & 0.51 & 0.11 & 0.36 & -0.11 & 0.15 & 0.01 & 0.92 \\
\hline Triglycerides & -0.04 & 0.62 & 0.16 & 0.21 & 0.07 & 0.37 & 0.31 & 0.009 & -0.13 & 0.09 & -0.05 & 0.62 & 0.03 & 0.72 & 0.1 & 0.41 \\
\hline Total cholesterol & 0.14 & 0.07 & 0.08 & 0.54 & -0.01 & 0.87 & 0.1 & 0.42 & -0.16 & 0.03 & 0.03 & 0.81 & -0.03 & 0.71 & 0.04 & 0.14 \\
\hline
\end{tabular}

*Significant $p$ values after applying the Bonferroni correction. Note: BMI. Body mass index.

$\mathrm{p}=0.21)$. However, a positive correlation between this ratio and QUICKI was observed [HOMA $(\mathrm{r}=-0.228$, $\mathrm{p}=0.10)$; QUICKI $(\mathrm{r}=0.277, \mathrm{p}=0.04)]$ in females.

\section{Discussion}

In acquired or genetic lipodystrophies, abnormal fat distribution per se is a strong determinant of IR and metabolic alterations, and the loss of fat likely precedes the metabolic complications [21-24]. Increased amounts of visceral fat accompanied by decrease in peripheral adipose tissue are associated with an increase of lipolysis and free fat acid fluxes, which together can alter adipocytokine production [25].

Regarding body fat composition, our data showed that there were differences in body fat distribution between HIV-infected patients and HIV-uninfected controls, males and females with HIV-infection and patients with or without FMR-L despite similar BMI.

Actually, HIV-infected patients had less subcutaneous adipose tissue, females had a higher fat mass and men had higher VAT and VAT/SAT ratio. FMR-L patients had less subcutaneous adipose tissue and more visceral fat mass.

\section{Leptin}

Leptin levels were lower in HIV-infected patients namely in those with FMR-L, pointing to an association with body fat, demonstrated by the significant positive correlation between leptin and body circumferences, BMI, body fat mass evaluated by DXA and by CT scan. As previously shown, we also observed that leptin concentrations were higher in HIV-infected women compared to HIV-infected men and, therefore, the leptin sexual dimorphism remains unchanged in HIV infection [26-28]. In genetic lipodystrophy syndromes, very low plasma concentrations of leptin were observed when fat mass was profoundly reduced [29] and that correlated positively with total adiposity [30].

In our sample, HIV-infected patients with isolated central fat accumulation and mixed forms of lipodystrophy had higher BMI and leptin levels. Leptin levels were lower in those with isolated lipoatrophy, positively proportional to BMI. Most studies, that did not differentiated between patients with predominant lipoatrophy, lipohypertrophy, and mixed patterns of fat redistribution, have failed to find significantly lower leptin levels in subjects with HIV lipodystrophy [24,31,32], but when subjects with distinctive lipoatrophy have been studied separately, lower leptin levels in this subgroup of HIV lipodystrophy patients has been observed. Also, significant associations between leptin and subcutaneous fat has been reported and decreased subcutaneous fat correlated with reduced leptin levels after controlling for visceral fat quantity [33]. Total and regional adiposity were strong and positively associated with leptin in healthy controls and in HIV-infected patients, both in men and women, suggesting that adiposity remains the major determinant of plasma leptin levels in HIV infection [30]. In a prospective study, there was a significant positive correlation between the appearance of lipoatrophy and the leptin levels [32,34].

In our sample, leptin was positive and significantly associated with fasting and 2 hours insulin on OGTT and HOMA in patients with FMR-L, and glucose and insulin 
Table 6 Associations between adipokines and anthropometric and metabolic parameters in patients with and without FMR defined lipodystrophy

\begin{tabular}{|c|c|c|c|c|c|c|c|c|c|c|}
\hline \multirow{4}{*}{ Correlation between } & \multicolumn{2}{|c|}{ Leptin } & \multicolumn{2}{|c|}{ Resistin } & \multicolumn{2}{|c|}{ PAI-1 } & \multicolumn{2}{|c|}{ TNF-a } & \multicolumn{2}{|c|}{ Adiponectin } \\
\hline & Without L & With L & Without L & With L & Without L & With L & Without L & With L & Without L & With L \\
\hline & $n=96$ & $n=70$ & $n=96$ & $\mathrm{n}=74$ & $\mathrm{n}=\mathbf{2 8}$ & $\mathrm{n}=\mathbf{2 5}$ & $n=101$ & $n=73$ & $\mathrm{n}=99$ & $\mathrm{n}=75$ \\
\hline & $r$ & $r$ & $r$ & $r$ & $r$ & $r$ & $r$ & $r$ & $r$ & $r$ \\
\hline Age & 0.10 & -0.01 & -0.001 & -0.10 & -0.09 & 0.25 & 0.12 & -0.19 & 0.04 & -0.12 \\
\hline Duration of HIV-infection & -0.06 & 0.38 & -0.01 & 0.23 & 0.18 & -0.11 & 0.11 & 0.11 & 0.13 & 0.16 \\
\hline Duration of CART & -0.24 & 0.31 & 0.04 & 0.14 & 0.12 & -0.16 & 0.05 & 0.05 & 0.04 & 0.21 \\
\hline Wais/hip ratio & 0.12 & 0.15 & 0.05 & 0.02 & 0.10 & 0.14 & 0.15 & -0.01 & 0.06 & -0.19 \\
\hline Thigh circumf. & $0.48^{*}$ & -0.02 & -0.17 & -0.12 & -0.004 & 0.06 & -0.06 & -0.02 & 0.07 & 0.07 \\
\hline Cervical circumf. & -0.06 & -0.24 & -0.02 & -0.18 & 0.17 & 0.11 & 0.21 & -0.03 & -0.08 & -0.08 \\
\hline Waist circumf. & $0.53^{*}$ & 0.20 & -0.11 & 0.03 & -0.06 & 0.18 & 0.08 & -0.01 & -0.19 & -0.19 \\
\hline Hip circumf. & $0.65^{*}$ & 0.15 & -0.20 & -0.03 & -0.07 & 0.06 & -0.04 & -0.04 & -0.04 & -0.04 \\
\hline Arm circumf. & $0.47^{*}$ & -0.06 & -0.02 & -0.21 & -0.13 & 0.12 & 0.01 & -0.21 & -0.07 & -0.07 \\
\hline CD4 cell count & -0.09 & 0.02 & -0.01 & -0.11 & 0.29 & 0.21 & 0.03 & 0.02 & -0.09 & -0.12 \\
\hline BMI & $0.66^{*}$ & 0.17 & -0.19 & -0.09 & -0.007 & 0.04 & 0.08 & -0.02 & -0.03 & -0.13 \\
\hline Glucose at 0 & 0.07 & -0.03 & 0.15 & 0.01 & 0.08 & 0.09 & 0.22 & 0.002 & 0.12 & 0.02 \\
\hline Glucose at $2 \mathrm{~h}$ on OGTT & 0.25 & 0.12 & 0.12 & 0.12 & -0.20 & -0.12 & 0.06 & -0.02 & -0.02 & -0.06 \\
\hline Insulin at 0 & 0.17 & 0.31 & 0.05 & 0.22 & -0.10 & 0.03 & 0.09 & 0.12 & -0.08 & -0.12 \\
\hline Insulin at $2 \mathrm{~h}$ on OGTT & 0.36 & 0.39 & 0.06 & 0.17 & -0.21 & -0.32 & 0.11 & 0.19 & -0.08 & -0.14 \\
\hline HOMA & 0.18 & 0.27 & 0.08 & 0.25 & -0.03 & 0.03 & 0.11 & 0.11 & -0.06 & -0.11 \\
\hline A1c & 0.15 & 0.008 & 0.19 & 0.11 & -0.34 & -0.02 & 0.24 & 0.00 & 0.14 & -0.02 \\
\hline Triglycerides & -0.004 & 0.03 & 0.06 & 0.17 & -0.17 & -0.18 & -0.13 & -0.17 & 0.08 & -0.03 \\
\hline Total cholesterol & 0.25 & -0.01 & -0.01 & -0.06 & -0.29 & 0.09 & 0.18 & -0.17 & -0.13 & 0.09 \\
\hline Total fat mass DXA \% & $0.877^{*}$ & $0.561^{*}$ & -0.14 & 0.07 & -0.05 & -0.05 & -0.07 & -0.12 & -0.17 & -0.19 \\
\hline Trunk fat mass DXA \% & $0.858^{*}$ & $0.547^{*}$ & -0.14 & 0.07 & -0.05 & 0.001 & -0.02 & -0.10 & -0.15 & -0.20 \\
\hline Leg fat mass DXA \% & $0.825^{*}$ & $0.464^{*}$ & -0.15 & 0.02 & -0.06 & -0.07 & -0.09 & -0.13 & -0.15 & -0.19 \\
\hline Arm fat mass DXA \% & $0.857^{*}$ & $0.650^{*}$ & -0.12 & 0.08 & -0.08 & -0.19 & -0.09 & -0.10 & -0.21 & -0.06 \\
\hline Total fat mass in $\mathrm{CT}$ & $0.774^{*}$ & $0.515^{*}$ & -0.19 & 0.15 & -0.07 & 0.07 & 0.13 & -0.04 & -0.08 & -0.14 \\
\hline VAT in CT & $0.483^{*}$ & 0.119 & -0.18 & 0.14 & 0.05 & 0.41 & 0.05 & -0.04 & -0.07 & -0.19 \\
\hline SAT in CT & $0.829^{*}$ & $0.587^{*}$ & -0.18 & 0.12 & -0.10 & -0.01 & 0.08 & -0.06 & -0.09 & -0.03 \\
\hline - & $-0.421^{*}$ & $-0.475^{*}$ & 0.10 & -0.04 & 0.04 & 0.31 & 0.002 & 0.07 & 0.003 & 0.004 \\
\hline
\end{tabular}

${ }^{*} p$ values $<0.001$ (significant applying the Bonferroni corretion).

at 2 hours on OGTT in patients without FMR-L. The role of leptin in the modulation of insulin sensitivity is complex. Extremely low levels of leptin in congenital generalized lipoatrophies were associated with severe IR, and significantly improved with replacement of leptin in mice and humans [35]. In conclusion, leptin levels appear to be primarily determined by total adiposity in HIV-infected individuals, independently of the presence of lipodystrophy [30].

\section{Adiponectin}

In our sample, adiponectin levels were higher in controls than in HIV-infected patients. Patients with no lipodystrophy had higher levels of adiponectin and patients with isolated central fat accumulation had the lowest levels of adiponectin. Contrary to other authors, who found higher adiponectin levels in HIV-infected women $[26,27]$, no such gender difference was observed in our patients, and no difference regarding the presence of FMR-L was also found. We found a significant negative correlation between adiponectin and total and trunk fat mass evaluated by DXA in the HIV-infected patients, and with arm fat mass in patients without FMR-L.

Kosmiski et al., observed that both total and regional adiposity were significant and negatively correlated with adiponectin levels in a control group, similar to other reports of general populations [30]. Also, these HIVinfected patients with leg SAT below the 10th percentile 
of controls had significantly lower adiponectin levels than controls with similar leg SAT volumes [30]. In some studies, no association between adiponectin and total adiposity was found in HIV-infected patients, while adiponectin was positively correlated with both extremity and abdominal SAT [36,37]. These relationships suggest that the remaining SAT in HIV-infected patients with moderate to severe lipoatrophy is dysfunctional rather than simply reduced in size, as for example, patients suffering from anorexia nervosa, a condition characterized by low body weight and severe global fat depletion (although not isolated subcutaneous adipose tissue), adiponectin levels are higher [38-40]. Findings of abnormal function or loss of adipose tissue in partial lipodystrophy syndromes (due to lamin A/C or to PPAR- $\gamma$ receptor mutations) and low adiponectinemia also support the hypothesis that low adiponectin concentrations in HIV-infected subjects with low levels of SAT are due to lipodystrophy per se [39,40]. Thus, lipodystrophies are characterized not only by a fat decreased but also by dysfunction or loss of adipocytes [30]. In HIV-infected men and women, with or without lipodystrophy, the normal negative relationship between visceral adiposity and adiponectin was maintained [30,36,37,41].

In HIV-infected patients, adiponectin levels correlated significantly with the percentage of limbs fat and insulin sensitivity $[24,42]$, although some authors state that adiponectin predicts insulin sensitivity independently of the amount and repartition of body fat [28], no such association was found between adiponectin and markers of IR in our sample.

It has been reported that in HIV lipodystrophic patients, adiponectin levels were significantly lower in patients with fat redistribution (and correlated inversely with serum triglycerides). The lowest levels were in peripheral lipoatrophic and central lipohypertrophic patients playing a central role in glucose, lipid metabolism and IR in these patients $[28,37,43,44]$.

\section{Ratio adiponectin/leptin}

Adiponectin/leptin ratio is considered to be a better predictor of insulin sensitivity and cardiovascular risk, and is usually considered independent of body mass. Adiponectin/leptin ratio has been negatively associated with IR, metabolic defects and cardiovascular risk markers [28]. We also found a positive correlation between this ratio and QUICKI index in women. This ratio was higher in patients with no lipodystrophy and lower in patients with isolated central fat accumulation, followed by the patients with mixed forms of lipodystrophy.

\section{Resistin}

Elevated resistin levels have been observed in obese and diabetic subjects, and increased resistin has been associated with IR in lean and obese subjects [45]. However, other studies found no association between circulating resistin levels and IR [46,47].

In our data, resistin was higher in controls and no significant differences were found in the four categories of body composition, or according to gender and presence of FMR-L in HIV-infected patients, similar to previously published data [48]. However, others found that resistin plasma levels were higher in lipodystrophic patients, being highest in those with isolated lipoatrophy [49]. Resistin was positively associated with duration of HIV-infection and with HOMA in patients with FMR-L, and negatively with hip circumference. Some authors previously demonstrated a correlation between resistin plasma levels and insulin resistance in lipodystrophic HIV patients [50] and others in HIV patients either with or without lipodystrophy [49].

TNF- $\boldsymbol{a}$

HIV lipodystrophy, familial partial lipodystrophy and obesity have been associated with high circulating levels of TNF- $\alpha[39,51,52]$. In our sample, no significant differences in TNF- $\alpha$ levels were observed between controls and HIV-infected patients, in patients with or without FMR-L or regarding the four categories of fat composition. However, we found a positive correlation between TNF- $\alpha$ and fasting glucose and A1c in patients without FMR-L.

TNF- $\alpha$ homeostasis is profoundly altered by HIV-1 infection itself [53] and it has been shown that HIV-1 infected patients have elevated pre-treatment levels of TNF- $\alpha$ but these levels dramatically decrease when cART is initiated [54]. In our sample, patients have been treated for a long period of time (maximum 20 years) and this could potentially explain similar levels in controls and HIV-infected patients. However, other authors showed that concentrations of the components of TNF- $\alpha$ system are not normalized when cART is effective. We did not observe any correlation between TNF- $\alpha$ and adiponectin levels $(\mathrm{r}=0.145 ; \mathrm{p}=0.06)$ although that has previously been described [54].

\section{PAI-1}

Patients with no lipodystrophy and mixed forms of lipodystrophy had higher levels of PAI-1, placing them at substantial risk of cardiovascular disease via impaired fibrinolysis (54). In our sample, the lowest levels of PAI1 were found in those with isolated central fat accumulation. No differences in PAI-1 levels between patients with or without FMR-L and according to gender were found, but a significant positive association between PAI-1 and VAT was observed in patients with FMR-L.

PAI-1 concentrations are increased in conditions characterized by increased VAT or SAT, hypertriglyceridemia, and hyperinsulinemia. Both VAT and SAT produce PAI-1, but data are controversial as to whether VAT produces 
more, similar, or less amounts of PAI-1 than SAT. Also, it has been suggested that PAI-1 originates from stromal cells in adipose tissue rather than from adipocytes. Patients with cART-associated lipodystrophy had elevated plasma PAI-1 concentrations [55]. HIV infected patients with fat redistribution had significant elevations in PAI-1, being waist-to-hip ratio the only significant predictor of PAI-1 [56].

Contrary to our data, $\mathrm{He}$ et al., found significantly increased plasma levels of PAI-1 in patients with lipodystrophy. They also found a positive correlation between plasma PAI-1 level and BMI, waist circumference, VAT, plus an inverse correlation with the percentage of limb fat. In a stepwise multiple linear regression model, VAT turned out to be the main predictor of plasma PAI-1 [57].

\section{Ghrelin}

Ghrelin was higher in HIV-infected patients than in controls. Fasting levels of serum ghrelin has been negatively correlated with BMI, and it is decreased in obesity and increased in cachexia [58]. Contrary to our data, lower levels of ghrelin have been reported in HIV-infected lipodystrophic patients [5,59]. Falasca et al., observed higher levels of ghrelin in patients with hypertriglyceridemia, as well as a positive correlation between ghrelin and triglyceride levels in patients with hypertriglyceridemia. No such association between ghrelin and triglyceride levels were observed in our sample $(\mathrm{r}=0.064, \mathrm{p}=0.42)$. Falasca concluded that adipokines may have crucial roles in the pathogenesis of hypertriglyceridemia and, thus, monitoring of ghrelin levels could be important to identify subjects with high cardiovascular risk [58].

\section{Limitations}

Several limitations of this study should be noted. A crosssectional design impairs establishing causality between cART use, lipoatrophy, and alterations in adipokine levels. It is not possible to determine whether adipokine abnormalities are due to reduced hormone expression in adipose tissue, decreased differentiation or decreased adipose cell numbers. The body fat redistribution was assessed clinically and patients divided in 4 groups but this does not preclude that some patients categorized into one subset could in fact have minor subclinical changes in their fat distribution not detectable by physical examination.

\section{Some aspects of the current study should be highlighted}

The study was performed in a highly experienced unit in the assessment of metabolic and body fat abnormalities in HIV-infected patients; the clinical assessment of lipodystrophy was performed by the same investigator (PF); and objective definitions of lipodystrophy (Fat Mass Ratio by DXA), visceral and subcutaneous fat mass by CT were used.

\section{Conclusion}

Leptin levels were lower in patients with lipodystrophy, indicating its close relationship with total body fat. A significant positive correlation between leptin and HOMA in patients with lipodystrophy demonstrated the role of this adipokine in insulin resistance. Adiponectin was lower in patients with isolated central fat accumulation and negatively correlated with visceral adipose tissue. The adiponectin/leptin ratio was lower in patients with isolated central fat accumulation. Patients with mixed forms of lipodystrophy had higher levels of PAI-1. Resistin was positively associated with HOMA in patients with FMR-defined lipodystrophy. Patients with no lipodystrophy and mixed forms of lipodystrophy had higher levels of PAI-1 and the lowest levels of PAI-1 were found in those with isolated central fat accumulation. Ghrelin was higher in HIV-infected patients than in controls despite the two groups being BMI-matched. Our results add to the evidence on the complexity of the relationship between adipokines, hormones related to body composition, and insulin resistance in the HIV redistribution syndrome, with different degrees of peripheral fat atrophy and central fat accumulation.

\section{Competing interest}

All the authors declare that they have no competing interests.

\section{Authors' contributions}

PF conceived the study, participated in its design, in the acquisition of data and drafted the manuscript; DC conceived the study, participated in its design and drafted the manuscript; ACS performed the statistical analysis and revised critically the manuscript; AJM performed the $C T$ scan and reviewed the data; EM critically revised the manuscript; JP performed the DXA and reviewed the data; AS critically revised the manuscript and JLM revised the study design. All authors read and approved the final manuscript.

\section{Acknowledgements}

We thank Alberto Santos for performing the DXA measurements and Conceição Gonçalves, PharmD for performing the hormonal measurements. The EPI Porto cohort was funded by Fundação para a Ciência e Tecnologia PTDC/SAU-ESA/108315/2008.

\section{Funding}

Research Fellowship Dr. Manuel Almeida Ruas, Portuguese Society of Diabetology. Research Fellowship of the Portuguese Association for Clinical Study of AIDS. Research Grant to support doctoral studies in the area of HIV/AIDS, GlaxoSmithKline Health Sciences Foundation.

\section{Author details}

${ }^{1}$ Endocrinology Department, Hospital de São João and University of Porto Medical School, Alameda Hernâni Monteiro, 4200 Porto, Portugal.

${ }^{2}$ Department of Clinical Epidemiology, Predictive Medicine and Public Health, University of Porto Medical School, Porto, Portugal. ${ }^{3}$ University of Porto Institute of Public Health, Porto, Portugal. ${ }^{4}$ Radiology Department, Hospital de São João and University of Porto Medical School, Porto, Portugal.

${ }^{5}$ Department of Infectious Diseases, Hospital Clinic, University of Barcelona Medical School, Barcelona, Spain. ${ }^{6}$ Nuclear Medicine Department, Hospital de São João, Porto, Portugal. ${ }^{7}$ Infectious Diseases Department, Hospital de São João and University of Porto Medical School, Porto, Portugal.

Received: 12 September 2013 Accepted: 13 June 2014 Published: 23 June 2014 


\section{References}

1. Garg A: Acquired and inherited lipodystrophies. N Engl J Med 2004, 350(12):1220-1234.

2. Veloso S, Escote X, Ceperuelo-Mallafre V, Lopez-Dupla M, Peraire J, Vilades C, Domingo P, Castro A, Olona M, Sirvent JJ, Leal M, Vendrell J, Richart C, Vidal F: Leptin and adiponectin, but not IL18, are related with insulin resistance in treated HIV-1-infected patients with lipodystrophy. Cytokine 2012, 58(2):253-260

3. Ronti T, Lupattelli G, Mannarino E: The endocrine function of adipose tissue: an update. Clin Endocrinol (Oxf) 2006, 64(4):355-365.

4. Falasca K, Ucciferri C, Mancino P, Di lorio A, Vignale F, Pizzigallo E, Vecchiet J: Cystatin C, adipokines and cardiovascular risk in HIV infected patients. Curr HIV Res 2010, 8(5):405-410.

5. Koutkia P, Meininger G, Canavan B, Breu J, Grinspoon S: Metabolic regulation of growth hormone by free fatty acids, somatostatin, and ghrelin in HIV-lipodystrophy. Am J Physiol Endocrinol Metab 2004, 286(2):E296-E303.

6. Sweeney LL, Brennan AM, Mantzoros CS: The role of adipokines in relation to HIV lipodystrophy. AIDS 2007, 21(8):895-904.

7. Samaras K, Gan SK, Peake PW, Carr A, Campbell LV: Proinflammatory markers, insulin sensitivity, and cardiometabolic risk factors in treated HIV infection. Obesity (Silver Spring) 2009, 17(1):53-59.

8. Santos AC, Ebrahim S, Barros H: Gender, socio-economic status and metabolic syndrome in middle-aged and old adults. BMC Public Health 2008, 8:62.

9. CDC: Revised classification system for HIV infection and expanded surveillance case definition for AIDS among adolescents and adults. Recomm Rep 1993, 41:1-19.

10. Freitas P, Santos AC, Carvalho D, Pereira J, Marques R, Martinez E, Sarmento A, Medina JL: Fat mass ratio: an objective tool to define lipodystrophy in hiv-infected patients under antiretroviral therapy. J Clin Densitom 2010, 13(2):197-203

11. Freitas $P$, Carvalho D, Santos AC, Mesquita J, Correia F, Xerinda S, Marques $R$ Martinez E, Sarmento A, Medina JL: Assessment of body fat composition disturbances by bioimpedance analysis in HIV-infected adults. J Endocrinol Investig 2011, 34(10):e321-e329.

12. Freitas $P$, Carvalho D, Santos AC, Matos MJ, Madureira AJ, Marques R, Martinez E, Sarmento A, Medina JL: Prevalence of obesity and its relationship to clinical lipodystrophy in HIV-infected adults on anti-retroviral therapy. J Endocrinol Investig 2012, 35(11):964-970.

13. Freitas $P$, Carvalho D, Souto $S$, Santos AC, Xerinda S, Marques R, Martinez E, Sarmento A, Medina JL: Impact of Lipodystrophy on the prevalence and components of metabolic syndrome in HIV-infected patients. BMC Infect Dis 2011, 11:246.

14. Chobanian AV, Bakris GL, Black HR, Cushman WC, Green LA, Izzo JL Jr, Jones DW, Materson BJ, Oparil S, Wright JT Jr, Roccella EJ, National Heart, Lung Blood Institute Joint National Committee on Prevention, Detection Evaluation Treatment of High Blood, Pressure, National High Blood Pressure Education Program Coordinating, Committee: The Seventh Report of the Joint National Committee on Prevention, Detection, Evaluation, and Treatment of High Blood Pressure: the JNC 7 report. JAMA 2003, 289(19):2560-2572.

15. Alberti KG, Eckel RH, Grundy SM, Zimmet PZ, Cleeman Jl, Donato KA, Fruchart JC, James WP, Loria CM, Smith SC Jr: Harmonizing the metabolic syndrome: a joint interim statement of the International Diabetes Federation Task Force on Epidemiology and Prevention; National Heart, Lung, and Blood Institute; American Heart Association; World Heart Federation; International Atherosclerosis Society; and International Association for the Study of Obesity. Circulation 2009, 120(16):1640-1645.

16. Bonnet E, Delpierre C, Sommet A, Marion-Latard F, Herve R, Aquilina C, Labau E, Obadia M, Marchou B, Massip P, Perret B, Bernard J: Total body composition by DXA of 241 HIV-negative men and 162 HIV-infected men: proposal of reference values for defining lipodystrophy. J Clin Densitom 2005, 8(3):287-292.

17. van der Kooy K, Seidell JC: Techniques for the measurement of visceral fat: a practical guide. Int J Obes Relat Metab Disord 1993, 17(4):187-196.

18. Yoshizumi T, Nakamura T, Yamane M, Islam AH, Menju M, Yamasaki K, Arai T, Kotani K, Funahashi T, Yamashita S, Matsuzawa Y: Abdominal fat: standardized technique for measurement at CT. Radiology 1999 211(1):283-286
19. Matthews DR, Hosker JP, Rudenski AS, Naylor BA, Treacher DF, Turner RC: Homeostasis model assessment: insulin resistance and beta-cell function from fasting plasma glucose and insulin concentrations in man. Diabetologia 1985, 28(7):412-419.

20. Katz A, Nambi SS, Mather K, Baron AD, Follmann DA, Sullivan G, Quon MJ: Quantitative insulin sensitivity check index: a simple, accurate method for assessing insulin sensitivity in humans. J Clin Endocrinol Metab 2000, 85(7):2402-2410.

21. Grinspoon S: Insulin resistance in the HIV-lipodystrophy syndrome. Trends Endocrinol Metab 2001, 12(9):413-419.

22. Reitman ML, Arioglu E, Gavrilova O, Taylor SI: Lipoatrophy revisited. Trends Endocrinol Metab 2000, 11(10):410-416.

23. Hegele RA: Insulin resistance in human partial lipodystrophy. Curr Atheroscler Rep 2000, 2(5):397-404.

24. Mynarcik DC, Combs T, McNurlan MA, Scherer PE, Komaroff E, Gelato MC: Adiponectin and leptin levels in HIV-infected subjects with insulin resistance and body fat redistribution. J Acquir Immune Defic Syndr 2002, 31(5):514-520

25. Gougeon ML, Penicaud L, Fromenty B, Leclercq P, Viard JP, Capeau J: Adipocytes targets and actors in the pathogenesis of HIV-associated lipodystrophy and metabolic alterations. Antivir Ther 2004, 9(2):161-177.

26. Considine RV, Sinha MK, Heiman ML, Kriauciunas A, Stephens TW, Nyce MR, Ohannesian JP, Marco CC, McKee LJ, Bauer TL, Caro JF: Serum immunoreactive-leptin concentrations in normal-weight and obese humans. N Engl J Med 1996, 334(5):292-295.

27. Arita Y, Kihara S, Ouchi N, Takahashi M, Maeda K, Miyagawa J, Hotta K, Shimomura I, Nakamura T, Miyaoka K, Kuriyama H, Nishida M, Yamashita S, Okubo K, Matsubara K, Muraguchi M, Ohmoto Y, Funahashi T, Matsuzawa Y: Paradoxical decrease of an adipose-specific protein, adiponectin, in obesity. Biochem Biophys Res Commun 1999, 257(1):79-83.

28. Vigouroux C, Maachi M, Nguyen TH, Coussieu C, Gharakhanian S, Funahashi T, Matsuzawa Y, Shimomura I, Rozenbaum W, Capeau J, Bastard JP: Serum adipocytokines are related to lipodystrophy and metabolic disorders in HIV-infected men under antiretroviral therapy. AIDS 2003, 17(10):1503-1511.

29. Haque WA, Shimomura I, Matsuzawa Y, Garg A: Serum adiponectin and leptin levels in patients with lipodystrophies. J Clin Endocrinol Metab 2002, 87(5):2395.

30. Kosmiski LA, Bacchetti P, Kotler DP, Heymsfield SB, Lewis CE, Shlipak MG, Scherzer R, Grunfeld C: Relationship of fat distribution with adipokines in human immunodeficiency virus infection. J Clin Endocrinol Metab 2008, 93(1):216-224

31. Martini G, Valenti R, Giovani S, Campagna S, Franci B, Nuti R: Leptin and body composition in healthy postmenopausal women. Panminerva Med 2001, 43(3):149-154

32. Wunder D, Bersinger NA, Fux C, Weber R, Bernasconi E, Cavassini M, Bucher HC, Schiffer V, Schmid P. Furrer H: Plasma leptin levels in men are not related to the development of lipoatrophy during antiretroviral therapy. AIDS 2005, 19(16):1837-1842.

33. Koutkia P, Canavan B, Breu J, Johnson ML, Depaoli A, Grinspoon SK: Relation of leptin pulse dynamics to fat distribution in HIV-infected patients. Am J Clin Nutr 2004, 79(6):1 103-1109.

34. Freitas $P C D$, Santos $A C$, Xerinda $S$, Matos MJ, Marques R, Serrão R, Pereira Sarmento A, Medina JL: A one year prospective study indicates that HIV patients under antiretroviral therapy worsen lipodystrophy. Ara Bras Endocrinol Metabol 2008, 52:5945.

35. Oral EA, Simha V, Ruiz E, Andewelt A, Premkumar A, Snell P, Wagner AJ, DePaoli AM, Reitman ML, Taylor SI, Gorden P, Garg A: Leptin-replacement therapy for lipodystrophy. N Engl J Med 2002, 346(8):570-578.

36. Tong Q, Sankale JL, Hadigan CM, Tan G, Rosenberg ES, Kanki PJ, Grinspoon SK, Hotamisligil GS: Regulation of adiponectin in human immunodeficiency virus-infected patients: relationship to body composition and metabolic indices. J Clin Endocrinol Metab 2003, 88(4):1559-1564.

37. Addy CL, Gavrila A, Tsiodras S, Brodovicz K, Karchmer AW, Mantzoros CS: Hypoadiponectinemia is associated with insulin resistance, hypertriglyceridemia, and fat redistribution in human immunodeficiency virus-infected patients treated with highly active antiretroviral therapy. J Clin Endocrinol Metab 2003, 88(2):627-636.

38. Delporte ML, Brichard SM, Hermans MP, Beguin C, Lambert M: Hyperadiponectinaemia in anorexia nervosa. Clin Endocrinol (Oxf) 2003 58(1):22-29. 
39. Wong SP, Huda M, English P, Bargiotta A, Wilding JP, Johnson A, Corrall R, Pinkney $\mathrm{JH}$ : Adipokines and the insulin resistance syndrome in familial partial lipodystrophy caused by a mutation in lamin A/C. Diabetologia 2005, 48(12):2641-2649.

40. Savage DB, Tan GD, Acerini CL, Jebb SA, Agostini M, Gurnell M, Williams RL, Umpleby AM, Thomas EL, Bell JD, Dixon AK, Dunne F, Boiani R, Cinti S, Vidal-Puig A, Karpe F, Chatterjee VK, O'Rahilly S: Human metabolic syndrome resulting from dominant-negative mutations in the nuclear receptor peroxisome proliferator-activated receptor-gamma. Diabetes 2003, 52(4):910-917.

41. Kosmiski L, Kuritzkes D, Lichtenstein K, Eckel R: Adipocyte-derived hormone levels in HIV lipodystrophy. Antivir Ther 2003, 8(1):9-15.

42. Mynarcik DC, McNurlan MA, Steigbigel RT, Fuhrer J, Gelato MC: Association of severe insulin resistance with both loss of limb fat and elevated serum tumor necrosis factor receptor levels in HIV lipodystrophy. J Acquir Immune Defic Syndr 2000, 25(4):312-321.

43. Estrada V, Martinez-Larrad MT, Gonzalez-Sanchez JL, De Villar NG, Zabena C, Fernandez C, Serrano-Rios M: Lipodystrophy and metabolic syndrome in HIV-infected patients treated with antiretroviral therapy. Metabolism 2006, 55(7):940-945.

44. Chaparro J, Reeds DN, Wen W, Xueping E, Klein S, Semenkovich CF, Bae KT, Quirk EK, Powderly WG, Yarasheski KE, Li E: Alterations in thigh subcutaneous adipose tissue gene expression in protease inhibitorbased highly active antiretroviral therapy. Metabolism 2005, 54(5):561-567.

45. Silha JV, Krsek M, Skrha JV, Sucharda P, Nyomba BL, Murphy LJ: Plasma resistin, adiponectin and leptin levels in lean and obese subjects: correlations with insulin resistance. Eur J Endocrinol 2003, 149(4):331-335.

46. Lee JH, Chan JL, Yiannakouris N, Kontogianni M, Estrada E, Seip R, Orlova C, Mantzoros CS: Circulating resistin levels are not associated with obesity or insulin resistance in humans and are not regulated by fasting or leptin administration: cross-sectional and interventional studies in normal, insulin-resistant, and diabetic subjects. J Clin Endocrinol Metab 2003, 88(10):4848-4856.

47. Youn BS, Yu KY, Park HJ, Lee NS, Min SS, Youn MY, Cho YM, Park YJ, Kim SY, Lee HK, Park KS: Plasma resistin concentrations measured by enzymelinked immunosorbent assay using a newly developed monoclonal antibody are elevated in individuals with type 2 diabetes mellitus. J Clin Endocrinol Metab 2004, 89(1):150-156.

48. Barb D, Wadhwa SG, Kratzsch J, Gavrila A, Chan JL, Williams CJ, Karchmer AW, Mantzoros CS: Circulating resistin levels are not associated with fat redistribution, insulin resistance, or metabolic profile in patients with the highly active antiretroviral therapy-induced metabolic syndrome. J Clin Endocrinol Metab 2005, 90(9):5324-5328.

49. Escote $X$, Miranda M, Veloso S, Domingo P, Alonso-Villaverde C, Peraire J, Vilades C, Alba V, Olona M, Castro A, Lopez-Dupla M, Sirvent JJ, Vicente V, Vendrell J, Richart C, Vidal F, and HIV Lipodystrophy Study Group: Lipodystrophy and insulin resistance in combination antiretroviral treated HIV-1-infected patients: implication of resistin. J Acquir Immune Defic Syndr 2011, 57(1):16-23

50. Kamin D, Hadigan C, Lehrke M, Mazza S, Lazar MA, Grinspoon S: Resistin levels in human immunodeficiency virus-infected patients with lipoatrophy decrease in response to rosiglitazone. J Clin Endocrinol Metab 2005, 90(6):3423-3426

51. Johnson JA, Albu JB, Engelson ES, Fried SK, Inada Y, Ionescu G, Kotler DP Increased systemic and adipose tissue cytokines in patients with HIV-associated lipodystrophy. Am J Physiol Endocrinol Metab 2004, 286(2):E261-E271.

52. Hauner $\mathrm{H}$ : Secretory factors from human adipose tissue and their functional role. Proc Nutr Soc 2005, 64(2):163-169.

53. Vidal F, Domingo P, Vilades C, Peraire J, Arnedo M, Alcami J, Leal M, Villarroya F, Gatell JM: Pharmacogenetics of the lipodystrophy syndrome associated with HIV infection and combination antiretroviral therapy. Expert Opin Drug Metab Toxicol 2011, 7(11):1365-1382.

54. Domingo P, Vidal F, Domingo JC, Veloso S, Sambeat MA, Torres F, Sirvent Jر J, Vendrell J, Matias-Guiu X, Richart C, HIV-Frs Study Group: Tumour necrosis factor alpha in fat redistribution syndromes associated with combination antiretroviral therapy in HIV-1-infected patients: potential role in subcutaneous adipocyte apoptosis. Eur J Clin Investig 2005, 35(12):771-780.

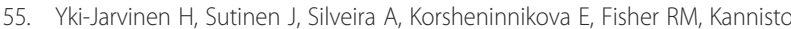
K, Ehrenborg E, Eriksson P, Hamsten A: Regulation of plasma PAI-1 concentrations in HAART-associated lipodystrophy during rosiglitazone therapy. Arterioscler Thromb Vasc Biol 2003, 23(4):688-694

56. Hadigan C, Meigs JB, Rabe J, D'Agostino RB, Wilson PW, Lipinska I, Tofler GH, Grinspoon SS: Increased PAI-1 and TPA antigen levels are reduced with metformin therapy in HIV-infected patients with fat redistribution and insulin resistance. J Clin Endocrinol Metab 2001, 86(2):939-943.

57. He G, Andersen O, Haugaard SB, Lihn AS, Pedersen SB, Madsbad S, Richelsen B: Plasminogen activator inhibitor type 1 (PAl-1) in plasma and adipose tissue in HIV-associated lipodystrophy syndrome. Implications of adipokines. Eur J Clin Invest 2005, 35(9):583-590.

58. Falasca K, Manigrasso MR, Racciatti D, Zingariello P, Dalessandro M, Ucciferri C, Mancino P, Marinopiccoli M, Petrarca C, Conti P, Pizzigallo E, Guagnano MT, Vecchiet J: Associations between hypertriglyceridemia and serum ghrelin, adiponectin, and IL-18 levels in HIV-infected patients. Ann Clin Lab Sci 2006, 36(1):59-66.

59. Rietschel P, Hadigan C, Corcoran C, Stanley T, Neubauer G, Gertner J, Grinspoon S: Assessment of growth hormone dynamics in human immunodeficiency virus-related lipodystrophy. J Clin Endocrinol Metab 2001, 86(2):504-510.

doi:10.1186/1471-2334-14-347

Cite this article as: Freitas et al:: Adipokines, hormones related to body composition, and insulin resistance in HIV fat redistribution syndrome. BMC Infectious Diseases 2014 14:347.

\section{Submit your next manuscript to BioMed Central and take full advantage of:}

- Convenient online submission

- Thorough peer review

- No space constraints or color figure charges

- Immediate publication on acceptance

- Inclusion in PubMed, CAS, Scopus and Google Scholar

- Research which is freely available for redistribution 